\title{
The cortical activation pattern by a rehabilitation robotic hand: a functional NIRS study
}

\section{Pyung-Hun Chang ${ }^{1}$, Seung-Hee Lee ${ }^{1}$, Gwang Min Gu ${ }^{2}$, Seung-Hyun Lee ${ }^{3}$, Sang-Hyun Jin ${ }^{3}$, Sang Seok Yeo ${ }^{4}$, Jeong Pyo Seo ${ }^{4}$ and Sung Ho Jang ${ }^{4}$ *}

'Department of Robotics Engineering, Graduate School, Daegu Gyeongbuk Institute of Science and Technology, Taegu, South Korea

2 Department of Mechanical Engineering, Graduate School, Korea Advance Institute of Science and Technology, Taegu, South Korea

${ }^{3}$ Robotics Research Division, Daegu Gyeongbuk Institute of Science and Technology, Taegu, South Korea

${ }^{4}$ Department of Physical Medicine and Rehabilitation, College of Medicine, Yeungnam University, Taegu, South Korea

\section{Edited by:}

Ulf Ziemann, Eberhard Karls University

Tübingen, Germany

Reviewed by:

Alireza Gharabaghi, University Hospital

Tuebingen, Germany

Ann-Christine Ehlis, University

Hospital Tuebingen, Germany

\section{*Correspondence:}

Sung Ho Jang, Department of Physical Medicine and Rehabilitation, College

of Medicine, Yeungnam University,

317-1 Daemyungdong, Namku,

Taegu 705-717, South Korea

e-mail:strokerehab@hanmail.net
Introduction: Clarification of the relationship between external stimuli and brain response has been an important topic in neuroscience and brain rehabilitation. In the current study, using functional near infrared spectroscopy (fNIRS), we attempted to investigate cortical activation patterns generated during execution of a rehabilitation robotic hand.

Methods: Ten normal subjects were recruited for this study. Passive movements of the right fingers were performed using a rehabilitation robotic hand at a frequency of 0.5 $\mathrm{Hz}$. We measured values of oxy-hemoglobin $(\mathrm{HbO})$, deoxy-hemoglobin $(\mathrm{HbR})$ and totalhemoglobin $(\mathrm{HbT})$ in five regions of interest: the primary sensory-motor cortex (SM1), hand somatotopy of the contralateral SM1, supplementary motor area (SMA), premotor cortex (PMC), and prefrontal cortex (PFC).

Results: $\mathrm{HbO}$ and $\mathrm{HbT}$ values indicated significant activation in the left SM1, left SMA, left PMC, and left PFC during execution of the rehabilitation robotic hand (uncorrected, $p<$ $0.01)$. By contrast, $\mathrm{HbR}$ value indicated significant activation only in the hand somatotopic area of the left SM1 (uncorrected, $p<0.01$ ).

Conclusions: Our results appear to indicate that execution of the rehabilitation robotic hand could induce cortical activation.

Keywords: functional NIRS, robot, cortical activation, brain plasticity, rehabilitation

\section{INTRODUCTION}

In recent decades, many rehabilitation robots have been developed for patients with brain injury (Aisen et al., 1997; Krebs et al., 1998; Volpe et al., 2000; Lum et al., 2002, 2006; Kahn et al., 2006; Masiero et al., 2007; Kwakkel et al., 2008; Rabadi et al., 2008; Housman et al., 2009; Lo et al., 2010; Conroy et al., 2011; Lapitskaya et al., 2011). These rehabilitation robots have been designed to aid in improvement or to assist with functional activity of patients with brain injury. The basic principle of brain rehabilitation is based on manipulation of external stimuli, which can induce activation of the cerebral cortex (Kaplan, 1988). Therefore, clarification of the relationship between external stimuli and brain response has been an important topic in neuroscience and brain rehabilitation. Likewise, regarding use of rehabilitation robots for patients with brain injury, elucidation of brain response by execution of rehabilitation robots would be important, however, little is known about cortical activation patterns induced by proprioceptive inputs during execution of rehabilitation robots (Blicher and Nielsen, 2009; Kamibayashi et al., 2009; Li et al., 2012).

Several functional neuroimaging techniques, including functional MRI (fMRI), Positron Emission Tomography, and functional near infrared spectroscopy (fNIRS) are available for use in brain activation studies by external stimuli (Frahm et al., 1993;
Miyai et al., 2001; Perrey, 2008; LaPointe et al., 2009; Mihara et al., 2010; Kim et al., 2011; Leff et al., 2011; Gagnon et al., 2012). Among these techniques, fMRI, which can be employed repeatedly, produces no ionizing radiation, and shows high spatial resolution, has been used most frequently (Frahm et al., 1993). However, it is sensitive to artifact resulting from motion and metallic materials. Robots are usually made of metal, and rehabilitation robots can perform large movements. Therefore, fMRI might not be appropriate for use in brain activation study of rehabilitation robots although some studies have reported cortical effects by magnetic resonance-compatible rehabilitation robots (Tsekos et al., 2007; Astrakas et al., 2012). By contrast, less sensitivity of fNIRS to motion artifact and metal material has been demonstrated; therefore, fNIRS could be more appropriate for use in research on brain activation study of rehabilitation robots (Arenth et al., 2007; Irani et al., 2007; Perrey, 2008; Mihara et al., 2010; Leff et al., 2011). In addition, compared with other functional neuroimaging techniques, the fNIRS has the advantage of applicability in more realistic day-to-day rehabilitation settings.

Many previous studies have reported on cortical activation by passive movements of a joint (Reddy et al., 2001; Radovanovic et al., 2002; Chang et al., 2009; Szameitat et al., 2012). According to these previous studies, passive movements induced cortical 
activation mainly in the primary sensorimotor cortex (SM1), and premotor cortex (PMC) and supplementary motor area (SMA) are also involved in processing of somatosensory input by passive movements (Reddy et al., 2001; Radovanovic et al., 2002; Chang et al., 2009). In addition, it has been shown that cortical activation patterns during passive movements were similar to that of active movements (Szameitat et al., 2012). In this study, we developed a rehabilitation robotic hand and hypothesized that performance of passive movements by our rehabilitation robotic hand can induce the proper amount of somatosensory stimulation to induce activation of the cerebral cortex, particularly the contralateral SM1, PMC and SMA.

In the current study, using fNIRS we attempted to investigate cortical activation patterns generated during execution of a rehabilitation robotic hand.

\section{SUBJECTS AND METHODS \\ SUBJECTS}

Ten healthy right-handed subjects ( 6 males, 4 females; mean age $27.8 \pm 2.5$ years, range 24-32) with no history of neurological, physical, or psychiatric illness were recruited for this study. All subjects were asked to complete a questionnaire in order to confirm that they had no history of neurological, physical, or psychiatric illness. They understood the purpose of the study and provided written, informed consent prior to participation. The study protocol was approved by our Institutional Review Board.

\section{THE REHABILITATION ROBOTIC HAND}

The rehabilitation robotic hand consists of two moving parts for four fingers (from second to fifth finger) and thumb (Figures 1A, B). The moving part for four fingers consists of a finger holder, four bar linkage, one actuator, and a force-torque sensor system, which is driven by a timing belt (Figure 1C). The finger holders are made with Velcro straps that allow the human's hand to follow the end point trajectory of the robot (Figure 1D). The grasping motion of the human's hand can be realized using four bar linkages, which are designed by imitating the trajectory of real grasping motion (Figure 1E). The moving part for the thumb consists of two wires, two pulleys, and a tension adjuster. It is directly connected to the moving part for four fingers by the cable driven system. It uses only 1 degree of freedom (DOF) for grasping motion. When the motor rotates positive $90^{\circ}$, it performs grasping motion, and rotation of negative $90^{\circ}$ results in performance of extension motion. Continuous action of the motor results in realization of repetitive flexion-extension motion. For real time control, using the linux distros Fedora 10 with linux kernel ver. 2.6.24 and Realtime Hardware Abstraction Layer (RTAI) ver. 3.7.1 systems, using an encoder and a Sensoray s626 board, we realized real time sensing control. For more precise control, we used time delay control. As a result, the rehabilitation robotic hand showed a position error of $0.1 \sim 1^{\circ}$.

All subjects were asked to sit comfortably on a chair in an upright position during conduct of the experiment. They were instructed to relax their hands maximally and not to move

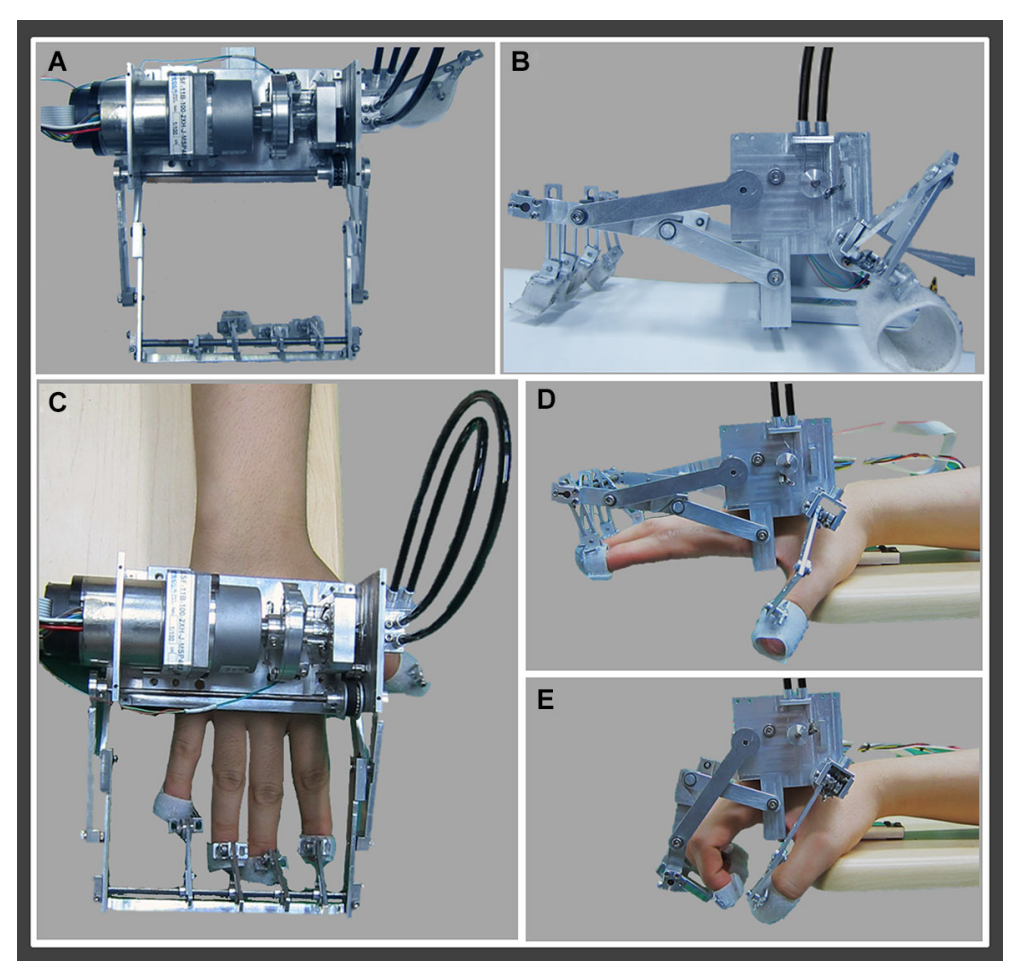

FIGURE 1 | Rehabilitation robotic hand. (A) Superior view of the rehabilitation robotic hand, (B) lateral view of the rehabilitation robotic hand, (C) fixed state of the right hand to the rehabilitation robotic hand, (D) finger extension (second to fifth fingers) and thumb abduction state by the rehabilitation robotic hand, (E) finger flexion (second to fifth fingers) and thumb adduction state by the rehabilitation robotic hand. 
the hand voluntarily or imagine the movements during performance of passive movements, which were executed by the robotic hand. The subject's right hand was fixed at the rehabilitation robotic hand. Using a block paradigm design (three cycles; resting (20 s)-movement by the robot (20 s)-resting (20 s)-movement by the robot (20 s)-resting (20 s)-movement by the robot (20s)) at a frequency of $0.5 \mathrm{~Hz}$, flexion-extension movements of the right fingers (from second to fifth) and abduction-adduction of the right thumb were performed by the rehabilitation robotic hand. During performance of passive hand movements, one experimenter confirmed that there had been no movement of the shoulder, elbow and wrist, and another experimenter observed changes in cortical activities using the monitor screen of the fNIRS system on a real-time basis (Yu et al., 2011; Dinomais et al., 2013).

\section{FUNCTIONAL NEAR INFRARED SPECTROSCOPY (NIRS)}

The fNIRS system (FOIRE-3000; Shimadzu, Kyoto, Japan), with continuous wave laser diodes with wavelengths of 780, 805, and $830 \mathrm{~nm}$, was used for recording of cortical activity at a sampling rate of $10 \mathrm{~Hz}$; we employed a 49-channel system with 30 optodes (15 light sources and 15 detectors). Based on the modified Beer-Lambert law, we acquired values for oxy-hemoglobin ( $\mathrm{HbO})$, deoxy-hemoglobin ( $\mathrm{HbR})$, and totalhemoglobin (HbT: mmol) following changes in levels of cortical concentration (Cope and Delpy, 1988). The international 10/20 system, with cranial vertex $(\mathrm{Cz})$ located beneath the 18 th channel, between the fourth light source and the seventh detector, was used for positioning of optodes; locations of the nasion, left ear, and right ear were identified in each subject. A stand-alone application was used for spatial registration of the 49 acquired channels on the Montreal Neurological Institute (MNI) brain based on locations of the nasion, left ear, and right ear, and the 18th channel on the $\mathrm{Cz}$ (Ye et al., 2009).

The software package NIRS-SPM ${ }^{1}$ implemented in the MATLAB environment (The Mathworks, USA) was used in analysis of fNIRS data. Gaussian smoothing with a full width at a half maximum (FWHM) of $2 \mathrm{~s}$ was applied to correction of noise from the fNIRS system (Ye et al., 2009). The wavelet minimum description length (MDL) based detrending algorithm was used for correction of signal distortion due to breathing or movement of the subject and general linear model (GLM) analysis with a

\footnotetext{
${ }^{1}$ http://bisp.kaist.ac.kr/NIRS-SPM
}

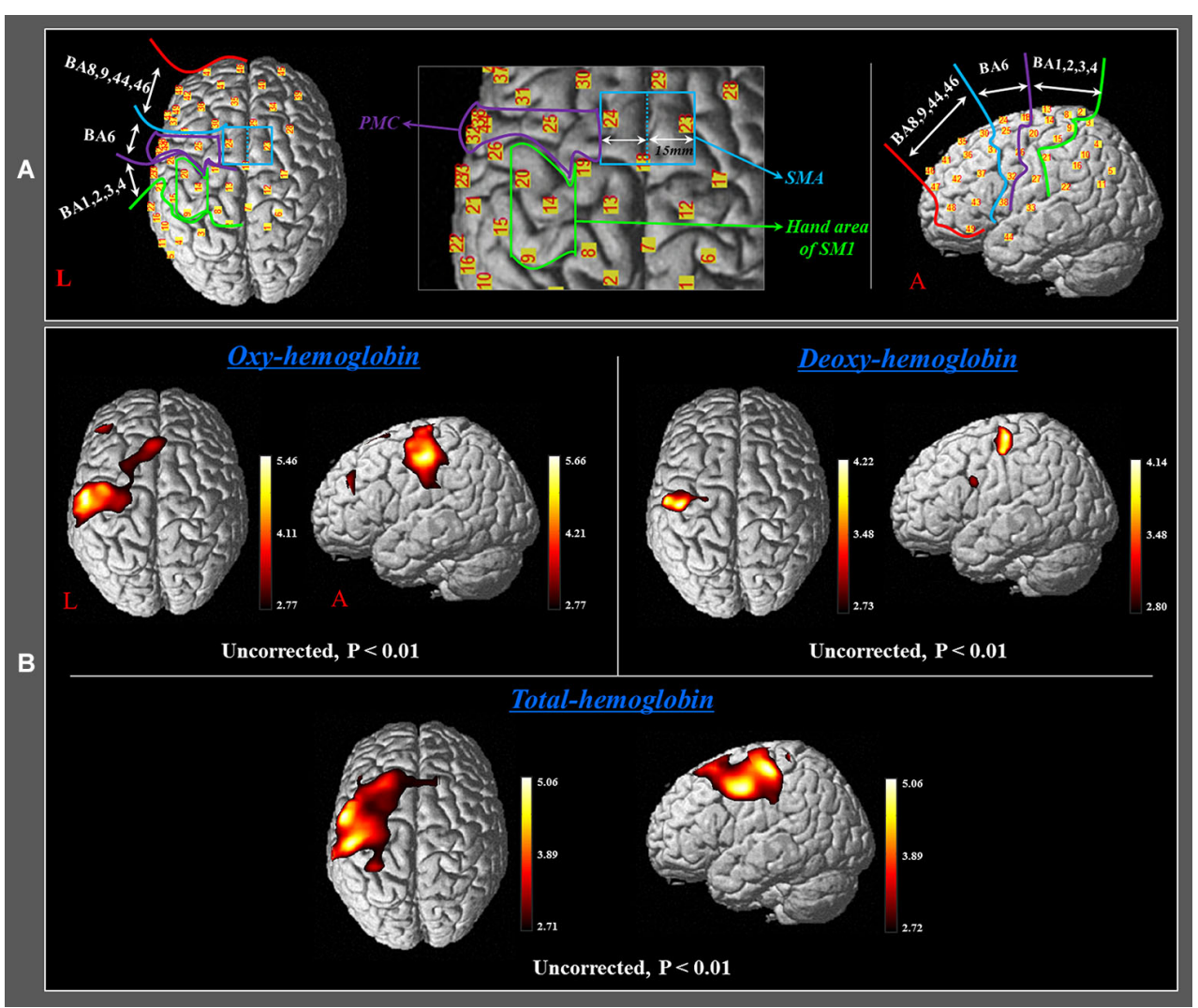

FIGURE 2 | (A) Four regions of interest based on the Brodmann area (BA) and anatomical location of areas of the brain. The primary sensory-motor cortex (SM1): BA 1, 2, 3, and 4; premotor cortex (PMC): BA 6 (BA 6, except for the SMA); supplementary motor area (SMA) (anterior boundary: vertical line to the anterior commissure, posterior boundary: anterior margin of M1, medial boundary: midline between the right and left hemispheres, lateral boundary: the line $15 \mathrm{~mm}$ lateral from the midline between the right and left hemispheres); prefrontal cortex (PFC): BA 8, 9, 44, 45, and 46. (B) Group-average activation map of $\mathrm{HbO}, \mathrm{HbR}$, and $\mathrm{HbT}$ during performance of passive movements of the right fingers, which were executed by the rehabilitation robotic hand using NIRS-SPM (uncorrected, $p<0.01$ ). 
canonical hemodynamic response curve was then performed in order to model the hypothesized $\mathrm{HbO}$ response under the experimental condition (Ye et al., 2009). Statistical parametric mapping (SPM) $t$-statistic maps were computed for group analysis, and, for stricter analysis, $\mathrm{HbO}, \mathrm{HbR}$, and $\mathrm{HbT}$ were considered significant at an uncorrected threshold of $p<0.01$ (Ye et al., 2009).

In order to investigate the cortical changing aspects of $\mathrm{HbO}$, $\mathrm{HbR}$ and $\mathrm{HbT}$ during performance of finger movements, which were executed by the rehabilitation robotic hand, we selected five regions of interest (ROI) based on the Brodmann area (BA) and anatomical locations of brain areas: SM1 (BA 1, 2, 3, and 4), the hand somatotopic area of the SM1 (medial boundary: medial margin of the precentral knob, lateral boundary: lateral margin of the precentral knob), PMC (BA 6, except for the SMA), SMA (anterior boundary: vertical line to the anterior commissure, posterior boundary: anterior margin of primary motor cortex (M1), medial boundary: midline between the right and left hemispheres, lateral boundary: the line $15 \mathrm{~mm}$ lateral from the midline between the right and left hemispheres), and the prefrontal cortex (PFC) (BA 8, 9, 44, 46) (Figure 2A; Afifi and Bergman, 2005; Mayka et al., 2006).

\section{RESULTS}

During performance of passive movements of the right fingers, which were executed by the rehabilitation robotic hand, a significant increase of $\mathrm{HbO}$ and $\mathrm{HbT}$ values was observed for both the left SM1 and the left SMA (uncorrected, $p<0.01$ ). In addition, a significant increase of $\mathrm{HbT}$ value was also observed for the left PMC and PFC during performance of passive movements of the right fingers. By contrast, in terms of $\mathrm{HbR}$ value, only the hand somatotopic area of the left SM1 showed a significant decrease, compared with other ROIs (uncorrected, $p<0.01$ ) (Figure 2B). In the time-course of actual hemodynamic responses, increased $\mathrm{HbO}$ and decreased $\mathrm{HbR}$ values were observed in ROI on the SM1 during performance of passive movements of the right fingers, which were executed by the rehabilitation robotic hand, and decreased or increased during rest phases (Figure 3).

\section{DISCUSSION}

$\mathrm{HbO}, \mathrm{HbR}$ and $\mathrm{HbT}$, which measure neural activity indirectly by detecting hemodynamic changes of the underlying cerebral cortex, are the most commonly used parameters of fNIRS (Irani et al., 2007; Perrey, 2008). The rationale for use of these parameters is based on the concept that neural activation in response to a stimulus results in increased energy demands in the activated area; consequently, an increase of $\mathrm{HbO}$ and concomitant decrease of $\mathrm{HbR}$ in the activated area occurs during neural activation (Perrey, 2008; Leff et al., 2011). In the current study, we investigated change of $\mathrm{HbO}, \mathrm{HbR}$, and $\mathrm{HbT}$ in five ROIs (SM1, hand somatotopy of the SM1, PMC, SMA, and PFC) during performance of passive movements of the fingers, which were executed by the rehabilitation robotic hand. With regard to $\mathrm{HbO}$ and $\mathrm{HbT}$ values in each of the five ROIs, we observed cerebral activation in the hand somatotopy of the contralateral SM1 and total SM1, contralateral PMC and SMA and contralateral PFC. In terms of $\mathrm{HbR}$ value, we observed cerebral activation only in the hand somatotopy of the contralateral SM1. On the other hand, the hemodynamic responses in ROI on the SM1 showed irregular fluctuation during the rest phases, as shown in Figure 3. These results might be related to repetitive stimulation to the cortical area or effects of nonlinear neurovascular coupling (Hudetz et al., 1992; Mayhew et al., 1996; Toronov et al., 2000).

Our rehabilitation robotic hand appears to work for the brain mainly via proprioceptive input by passive movements of fingers. This proprioceptive input is the sense for the change of the relative position of the hand and strength of passive movements, and is generated from millions of sensory receptors in skin, muscles, joints, and ligaments. Since introduction of functional neuroimaging techniques, many studies have reported activation of both the primary motor cortex (M1) and primary somatosensory cortex (S1) as a result of passive movements (Mima et al., 1999; Radovanovic et al., 2002; Francis et al., 2009; Blatow et al., 2011; Lee et al., 2012). Recently, using fMRI, Szameitat et al. (2012) reported that passive movements of wrist joint induced cortical activation in the SM1and SMA, and this cortical activation pattern was similar to that of active movements of wrist joint (Szameitat et al., 2012). However, training with active movements induces more significant improvements in motor performance with facilitation of cortical networks, compared with passive movements (Lotze et al., 2003; Perez et al., 2004).

Therefore, we believe that our results indicating activation of the SM1 by the rehabilitation robotic hand are consistent with those reported in previous studies. The pathway of M1 activation by somatosensory stimulation has not been clearly elucidated. Previously, it was thought that an afferent input arrives at the M1 through the S1 (Forss et al., 1994). However, there is general agreement that the M1 receives somatosensory input directly from the thalamus or dorsal column (Desmedt and Cheron, 1980; Dinner et al., 1987; Canedo, 1997; Jang et al., 2012). In addition, results of animal studies have demonstrated direct involvement of somatosensory input to the motor cortex in execution of voluntary movements (Asanuma and Arissian, 1984; Favorov et al., 1988), and branching axons to dorsal column nuclei were observed in the corticospinal tract (Bentivoglio and Rustioni, 1986; Martinez et al., 1995; Steward et al., 2004). However, in the current study, except for the contralateral SM1, the secondary motor area, including contralateral SMA and contralateral PMC, were activated by execution of our rehabilitation robotic hand. The secondary motor area is known to receive somatosensory input directly and this appears to be the basic mechanism of activation of the secondary motor area; likewise, activation of the SM1 (Hummelsheim et al., 1988; Rouiller et al., 1999; Chung et al., 2005; Kishi et al., 2009).

Although many rehabilitation robots have been developed for patients with brain injury, studies on cortical activation during execution of the robots are limited (Aisen et al., 1997; Krebs et al., 1998; Volpe et al., 2000; Lum et al., 2002, 2006; Kahn et al., 2006; Masiero et al., 2007; Kwakkel et al., 2008; Rabadi et al., 2008; Housman et al., 2009; Lo et al., 2010; Conroy et al., 2011; Lapitskaya et al., 2011). To the best of our knowledge, only a few studies using transcranial magnetic stimulation or fNIRS have demonstrated the cerebral effect by rehabilitation robots (Blicher and Nielsen, 2009; Kamibayashi et al., 2009; Li et al., 2012). In 2009, using transcranial magnetic stimulation 


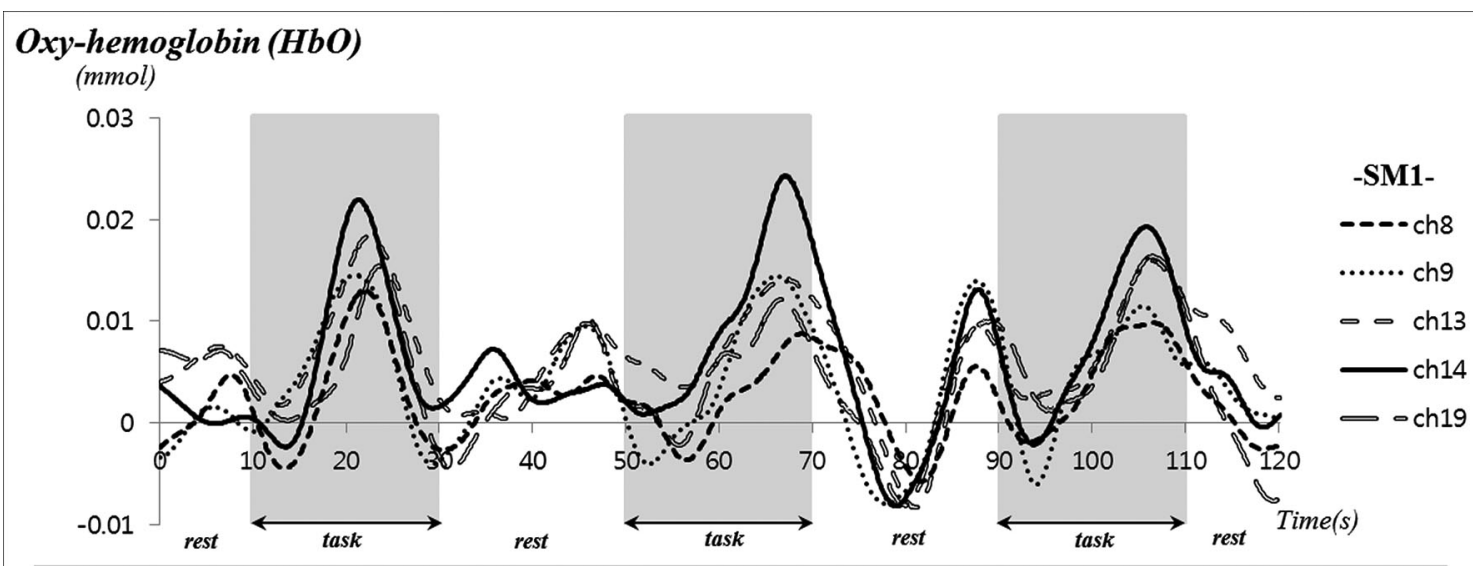

\section{Deoxy-hemoglobin (HbR)}

(mmol)

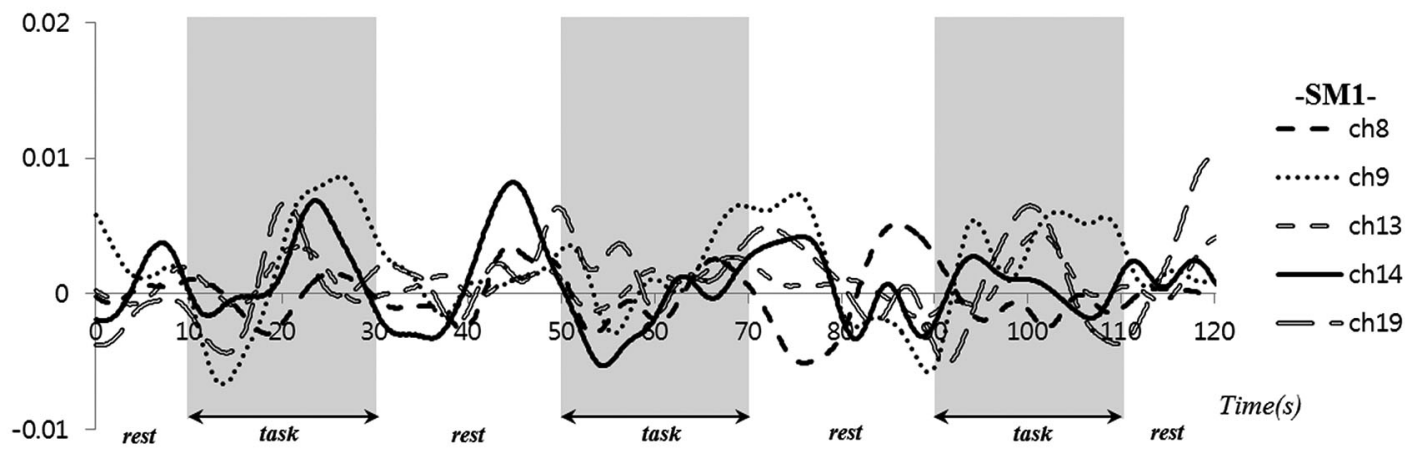

\section{Total-hemoglobin $(\mathrm{HbT})$}

(mmol)

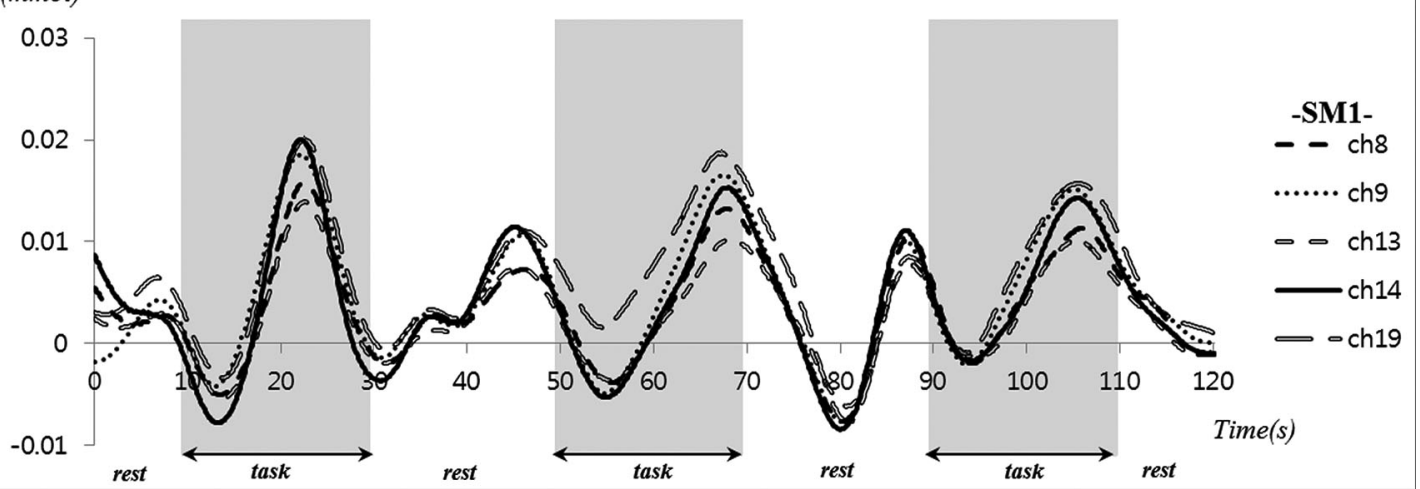

FIGURE 3 | Time course of hemodynamic responses for oxy-hemoglobin ( $\mathrm{HbO}$ ) deoxy-hemoglobin ( $\mathrm{HbR}$ ) and total-hemoglobin $(\mathrm{HbT})$ in region of interest on the sensory motor cortex during performance of passive movements of the right fingers, which were executed by the rehabilitation robotic hand in a subject (a 28-year-old male). in 13 normal subjects, Blicher and Nielsen investigated the cortical effect of robotic gait training using a driven gait orthosis (Blicher and Nielsen, 2009). They found that the decrease in short-interval intracortical inhibition after passive training in this gait robot may reflect a decrease in intracortical GABA activity, which could aid in acquisition of new motor skills. During the same year, Kamibayashi et al. investigated change of corticospinal excitability to the lower limb muscles using transcranial magnetic stimulation and transcranial electrical stimulation of the motor cortex while 13 normal subjects stepped passively in a robotic driven-gait orthosis (Kamibayashi et al., 2009). According to their findings, corticospinal excitability to the lower limb muscle was facilitated by load-related afferent inputs. In a recent study, Li et al. (2012), who developed a motion-tracking training robot by elbow flexion-extension movements, demonstrated the effect of this robot using fNIRS in 14 normal subjects (Li et al., 2012). They 
reported an increase in motion tracking precision and cortical activation in motor-control-related regions (SM1, SMA, PMC, and somatosensory areas) following motion-tracking training. In addition, they observed that, in terms of cortical activation, bimanual training was better than single-limb training.

In conclusion, we investigated cortical activation patterns during execution of our rehabilitation robotic hand; according to our results, the contralateral SM1, along with the contralateral PMC, contralateral SMA, and contralateral PFC were activated. Our results appear to suggest that execution of the rehabilitation robotic hand could induce cortical activation; therefore, we believe that our results would be helpful in research on development of rehabilitation robots. In addition, fNIRS could be a useful tool in research on the cortical effect of rehabilitation robots. For the clinical application, conduct of further studies on the optimal conditions for cortical activation and robot-assisted rehabilitation therapy by decoding of movement intention will be necessary (Gomez-Rodriguez et al., 2011). In addition, further studies on the training effect of this robotic hand in normal subjects and the clinical effect for patients with brain injury are also encouraged. However, the limitation that we did not monitor passive movements using an electromyographic method should be considered.

\section{ACKNOWLEDGMENTS}

This work was supported by the DGIST R\&D Program of the Ministry of Education, Science and Technology of Korea (14-BD0401).

\section{REFERENCES}

Afifi, A. K., and Bergman, R. A. (2005). Functional Neuroanatomy: Text and Atlas. New York: Lange Medical Books/McGraw-Hill.

Aisen, M. L., Krebs, H. I., Hogan, N., Mcdowell, F., and Volpe, B. T. (1997). The effect of robot-assisted therapy and rehabilitative training on motor recovery following stroke. Arch. Neurol. 54, 443-446. doi: 10.1001/archneur.1997. 00550160075019

Arenth, P. M., Ricker, J. H., and Schultheis, M. T. (2007). Applications of functional near-infrared spectroscopy (fNIRS) to neurorehabilitation of cognitive disabilities. Clin. Neuropsychol. 21, 38-57. doi: 10.1080/13854040600878785

Asanuma, H., and Arissian, K. (1984). Experiments on functional role of peripheral input to motor cortex during voluntary movements in the monkey. J. Neurophysiol. 52, 212-227.

Astrakas, L. G., Naqvi, S. H., Kateb, B., and Tzika, A. A. (2012). Functional MRI using robotic MRI compatible devices for monitoring rehabilitation from chronic stroke in the molecular medicine era (Review). Int. J. Mol. Med. 29, 963973. doi: 10.3892/ijmm.2012.942.

Bentivoglio, M., and Rustioni, A. (1986). Corticospinal neurons with branching axons to the dorsal column nuclei in the monkey. J. Comp. Neurol. 253, 260 276. doi: 10.1002/cne.902530212

Blatow, M., Reinhardt, J., Riffel, K., Nennig, E., Wengenroth, M., and Stippich, C. (2011). Clinical functional MRI of sensorimotor cortex using passive motor and sensory stimulation at 3 Tesla. J. Magn. Reson. Imaging 34, 429-437. doi: 10. 1002/jmri.22629

Blicher, J. U., and Nielsen, J. F. (2009). Cortical and spinal excitability changes after robotic gait training in healthy participants. Neurorehabil. Neural Repair 23, 143-149. doi: 10.1177/1545968308317973

Canedo, A. (1997). Primary motor cortex influences on the descending and ascending systems. Prog. Neurobiol. 51, 287-335. doi: 10.1016/s0301-0082(96)00058-5

Chang, M. C., Ahn, S. H., Cho, Y. W., Son, S. M., Kwon, Y. H., Lee, M. Y., et al. (2009). The comparison of cortical activation patterns by active exercise, proprioceptive input and touch stimulation in the human brain: a functional MRI study. NeuroRehabilitation 25, 87-92. doi: 10.3233/NRE-2009-0502
Chung, G. H., Han, Y. M., Jeong, S. H., and Jack, C. R. Jr. (2005). Functional heterogeneity of the supplementary motor area. AJNR Am. J. Neuroradiol. 26, 1819-1823.

Conroy, S. S., Whitall, J., Dipietro, L., Jones-Lush, L. M., Zhan, M., Finley, M. A., et al. (2011). Effect of gravity on robot-assisted motor training after chronic stroke: a randomized trial. Arch. Phys. Med. Rehabil. 92, 1754-1761. doi: 10. 1016/j.apmr.2011.06.016

Cope, M., and Delpy, D. T. (1988). System for long-term measurement of cerebral blood and tissue oxygenation on newborn infants by near infra-red transillumination. Med. Biol. Eng. Comput. 26, 289-294. doi: 10.1007/bf02447083

Desmedt, J. E., and Cheron, G. (1980). Central somatosensory conduction in man: neural generators and interpeak latencies of the far-field components recorded from neck and right or left scalp and earlobes. Electroencephalogr. Clin. Neurophysiol. 50, 382-403. doi: 10.1016/0013-4694(80)90006-1

Dinner, D. S., Luders, H., Lesser, R. P., and Morris, H. H. (1987). Cortical generators of somatosensory evoked potentials to median nerve stimulation. Neurology 37, 1141-1145. doi: 10.1212/wnl.37.7.1141

Dinomais, M., Chinier, E., Lignon, G., Richard, I., Ter Minassian, A., and Tich, S. N. (2013). The effect of video-guidance on passive movement in patients with cerebral palsy: fMRI study. Res. Dev. Disabil. 34, 3487-3496. doi: 10.1016/j.ridd. 2013.07.008

Favorov, O., Sakamoto, T., and Asanuma, H. (1988). Functional role of corticoperipheral loop circuits during voluntary movements in the monkey: a preferential bias theory. J. Neurosci. 8, 3266-3277.

Forss, N., Hari, R., Salmelin, R., Ahonen, A., Hamalainen, M., Kajola, M., et al. (1994). Activation of the human posterior parietal cortex by median nerve stimulation. Exp. Brain Res. 99, 309-315. doi: 10.1007/bf00239597

Frahm, J., Merboldt, K. D., and Hanicke, W. (1993). Functional MRI of human brain activation at high spatial resolution. Magn. Reson. Med. 29, 139-144. doi: $10.1002 / \mathrm{mrm} .1910290126$

Francis, S., Lin, X., Aboushoushah, S., White, T. P., Phillips, M., Bowtell, R., et al. (2009). fMRI analysis of active, passive and electrically stimulated ankle dorsiflexion. Neuroimage 44, 469-479. doi: 10.1016/j.neuroimage.2008. 09.017

Gagnon, L., Yucel, M. A., Dehaes, M., Cooper, R. J., Perdue, K. L., Selb, J., et al. (2012). Quantification of the cortical contribution to the NIRS signal over the motor cortex using concurrent NIRS-fMRI measurements. Neuroimage 59, 3933-3940. doi: 10.1016/j.neuroimage.2011.10.054

Gomez-Rodriguez, M., Peters, J., Hill, J., Scholkopf, B., Gharabaghi, A., and GrosseWentrup, M. (2011). Closing the sensorimotor loop: haptic feedback facilitates decoding of motor imagery. J. Neural. Eng. 8:036005. doi: 10.1088/17412560/8/3/036005

Housman, S. J., Scott, K. M., and Reinkensmeyer, D. J. (2009). A randomized controlled trial of gravity-supported, computer-enhanced arm exercise for individuals with severe hemiparesis. Neurorehabil. Neural Repair 23, 505-514. doi: $10.1177 / 1545968308331148$

Hudetz, A. G., Roman, R. J., and Harder, D. R. (1992). Spontaneous flow oscillations in the cerebral cortex during acute changes in mean arterial pressure. $J$. Cereb. Blood Flow Metab. 12, 491-499. doi: 10.1038/jcbfm. 1992.67

Hummelsheim, H., Bianchetti, M., Wiesendanger, M., and Wiesendanger, R. (1988). Sensory inputs to the agranular motor fields: a comparison between precentral, supplementary-motor and premotor areas in the monkey. Exp. Brain Res. 69, 289-298. doi: 10.1007/bf00247574

Irani, F., Platek, S. M., Bunce, S., Ruocco, A. C., and Chute, D. (2007). Functional near infrared spectroscopy (fNIRS): an emerging neuroimaging technology with important applications for the study of brain disorders. Clin. Neuropsychol. 21, 9-37. doi: 10.1080/13854040600910018

Jang, S. H., Kwon, Y. H., Lee, M. Y., Lee, D. Y., and Hong, J. H. (2012). Termination differences in the primary sensorimotor cortex between the medial lemniscus and spinothalamic pathways in the human brain. Neurosci. Lett. 516, 50-53. doi: 10.1016/j.neulet.2012.03.053

Kahn, L. E., Zygman, M. L., Rymer, W. Z., and Reinkensmeyer, D. J. (2006). Robot-assisted reaching exercise promotes arm movement recovery in chronic hemiparetic stroke: a randomized controlled pilot study. J. Neuroeng. Rehabil. 3:12. doi: 10.1186/1743-0003-3-12

Kamibayashi, K., Nakajima, T., Takahashi, M., Akai, M., and Nakazawa, K. (2009). Facilitation of corticospinal excitability in the tibialis anterior muscle during robot-assisted passive stepping in humans. Eur. J. Neurosci. 30, 100-109. doi: 10. 1111/j.1460-9568.2009.06795.x 
Kaplan, M. S. (1988). Plasticity after brain lesions: contemporary concepts. Arch. Phys. Med. Rehabil. 69, 984-991.

Kim, M. J., Hong, J. H., and Jang, S. H. (2011). The cortical effect of clapping in the human brain: a functional MRI study. NeuroRehabilitation 28, 75-79. doi: 10. 3233/NRE-2011-0634

Kishi, M., Sakakibara, R., Nagao, T., Terada, H., and Ogawa, E. (2009). Thalamic infarction disrupts spinothalamocortical projection to the mid-cingulate cortex and supplementary motor area. J. Neurol. Sci. 281, 104-107. doi: 10.1016/j.jns. 2009.03.012

Krebs, H. I., Hogan, N., Aisen, M. L., and Volpe, B. T. (1998). Robot-aided neurorehabilitation. IEEE Trans. Rehabil. Eng. 6, 75-87. doi: 10.1109/86.662623

Kwakkel, G., Kollen, B. J., and Krebs, H. I. (2008). Effects of robot-assisted therapy on upper limb recovery after stroke: a systematic review. Neurorehabil. Neural Repair 22, 111-121. doi: 10.1177/1545968307305457

Lapitskaya, N., Nielsen, J. F., and Fuglsang-Frederiksen, A. (2011). Robotic gait training in patients with impaired consciousness due to severe traumatic brain injury. Brain Inj. 25, 1070-1079. doi: 10.3109/02699052.2011.607782

LaPointe, K. E., Klein, J. A., Konkol, M. L., Kveno, S. M., Bhatt, E., Difabio, R. P., et al. (2009). Cortical activation during finger tracking vs. ankle tracking in healthy subjects. Restor. Neurol. Neurosci. 27, 253-264. doi: 10.3233/RNN-20090475

Lee, M. Y., Kim, S. H., Choi, B. Y., Chang, C. H., Ahn, S. H., and Jang, S. H. (2012). Functional MRI finding by proprioceptive input in patients with thalamic hemorrhage. NeuroRehabilitation 30, 131-136. doi: 10.3233/NRE-20120736

Leff, D. R., Orihuela-Espina, F., Elwell, C. E., Athanasiou, T., Delpy, D. T., Darzi, A. W., et al. (2011). Assessment of the cerebral cortex during motor task behaviours in adults: a systematic review of functional near infrared spectroscopy (fNIRS) studies. Neuroimage 54, 2922-2936. doi: 10.1016/j.neuroimage.2010. 10.058

Li, C., Inoue, Y., Liu, T., and Sun, L. (2012). Validation of bimanual-coordinated training supported by a new upper-limb rehabilitation robot: a near-infrared spectroscopy study. Disabil. Rehabil. Assist. Technol. doi: 10.3109/17483107. 2012.671439. [Epub ahead of print].

Lo, A. C., Guarino, P. D., Richards, L. G., Haselkorn, J. K., Wittenberg, G. F., Federman, D. G., et al. (2010). Robot-assisted therapy for long-term upper-limb impairment after stroke. N. Engl. J. Med. 362, 1772-1783. doi: 10.1056/NEJMoa 0911341

Lotze, M., Braun, C., Birbaumer, N., Anders, S., and Cohen, L. G. (2003). Motor learning elicited by voluntary drive. Brain 126, 866-872. doi: 10.1093/brain/ awg079

Lum, P. S., Burgar, C. G., Shor, P. C., Majmundar, M., and Van Der Loos, M. (2002). Robot-assisted movement training compared with conventional therapy techniques for the rehabilitation of upper-limb motor function after stroke. Arch. Phys. Med. Rehabil. 83, 952-959. doi: 10.1053/apmr.2001.33101

Lum, P. S., Burgar, C. G., Van Der Loos, M., Shor, P. C., Majmundar, M., and Yap, R. (2006). MIME robotic device for upper-limb neurorehabilitation in subacute stroke subjects: a follow-up study. J. Rehabil. Res. Dev. 43, 631-642. doi: 10. 1682/jrrd.2005.02.0044

Martinez, L., Lamas, J. A., and Canedo, A. (1995). Pyramidal tract and corticospinal neurons with branching axons to the dorsal column nuclei of the cat. Neuroscience 68, 195-206. doi: 10.1016/0306-4522(95)00133-4

Masiero, S., Celia, A., Rosati, G., and Armani, M. (2007). Robotic-assisted rehabilitation of the upper limb after acute stroke. Arch. Phys. Med. Rehabil. 88, 142-149. doi: 10.1016/j.apmr.2006.10.032

Mayhew, J. E., Askew, S., Zheng, Y., Porrill, J., Westby, G. W., Redgrave, P., et al. (1996). Cerebral vasomotion: a $0.1-\mathrm{Hz}$ oscillation in reflected light imaging of neural activity. Neuroimage 4, 183-193. doi: 10.1006/nimg.1996.0069

Mayka, M. A., Corcos, D. M., Leurgans, S. E., and Vaillancourt, D. E. (2006). Three-dimensional locations and boundaries of motor and premotor cortices as defined by functional brain imaging: a meta-analysis. Neuroimage 31, 14531474. doi: 10.1016/j.neuroimage.2006.02.004

Mihara, M., Yagura, H., Hatakenaka, M., Hattori, N., and Miyai, I. (2010). Clinical application of functional near-infrared spectroscopy in rehabilitation medicine. Brain Nerve 62, 125-132.

Mima, T., Sadato, N., Yazawa, S., Hanakawa, T., Fukuyama, H., Yonekura, Y., et al. (1999). Brain structures related to active and passive finger movements in man. Brain 122(Pt. 10), 1989-1997. doi: 10.1093/brain/122.10.1989
Miyai, I., Tanabe, H. C., Sase, I., Eda, H., Oda, I., Konishi, I., et al. (2001). Cortical mapping of gait in humans: a near-infrared spectroscopic topography study. Neuroimage 14, 1186-1192. doi: 10.1006/nimg.2001.0905

Perez, M. A., Lungholt, B. K., Nyborg, K., and Nielsen, J. B. (2004). Motor skill training induces changes in the excitability of the leg cortical area in healthy humans. Exp. Brain Res. 159, 197-205. doi: 10.1007/s00221-004-1947-5

Perrey, S. (2008). Non-invasive NIR spectroscopy of human brain function during exercise. Methods 45, 289-299. doi: 10.1016/j.ymeth.2008.04.005

Rabadi, M., Galgano, M., Lynch, D., Akerman, M., Lesser, M., and Volpe, B. (2008). A pilot study of activity-based therapy in the arm motor recovery post stroke: a randomized controlled trial. Clin. Rehabil. 22, 1071-1082. doi: 10. $1177 / 0269215508095358$

Radovanovic, S., Korotkov, A., Ljubisavljevic, M., Lyskov, E., Thunberg, J., Kataeva, G., et al. (2002). Comparison of brain activity during different types of proprioceptive inputs: a positron emission tomography study. Exp. Brain Res. 143, 276-285. doi: 10.1007/s00221-001-0994-4

Reddy, H., Floyer, A., Donaghy, M., and Matthews, P. M. (2001). Altered cortical activation with finger movement after peripheral denervation: comparison of active and passive tasks. Exp. Brain Res. 138, 484-491. doi: 10. 1007/s002210100732

Rouiller, E. M., Tanne, J., Moret, V., and Boussaoud, D. (1999). Origin of thalamic inputs to the primary, premotor and supplementary motor cortical areas and to area 46 in macaque monkeys: a multiple retrograde tracing study. J. Comp. Neurol. 409, 131-152. doi: 10.1002/(sici)1096-9861(19990621)409:1<131::aidcne10>3.0.co;2-a

Steward, O., Zheng, B., Ho, C., Anderson, K., and Tessier-Lavigne, M. (2004). The dorsolateral corticospinal tract in mice: an alternative route for corticospinal input to caudal segments following dorsal column lesions. J. Comp. Neurol. 472, 463-477. doi: 10.1002/cne.20090

Szameitat, A. J., Shen, S., Conforto, A., and Sterr, A. (2012). Cortical activation during executed, imagined, observed and passive wrist movements in healthy volunteers and stroke patients. Neuroimage 62, 266-280. doi: 10.1016/j. neuroimage.2012.05.009

Toronov, V., Franceschini, M. A., Filiaci, M., Fantini, S., Wolf, M., Michalos, A., et al. (2000). Near-infrared study of fluctuations in cerebral hemodynamics during rest and motor stimulation: temporal analysis and spatial mapping. Med. Phys. 27, 801-815. doi: 10.1118/1.598943

Tsekos, N. V., Khanicheh, A., Christoforou, E., and Mavroidis, C. (2007). Magnetic resonance-compatible robotic and mechatronics systems for image-guided interventions and rehabilitation: a review study. Annu. Rev. Biomed. Eng. 9, 351387. doi: 10.1146/annurev.bioeng.9.121806.160642

Volpe, B. T., Krebs, H. I., Hogan, N., Edelstein, O. L., Diels, C., and Aisen, M. (2000). A novel approach to stroke rehabilitation: robot-aided sensorimotor stimulation. Neurology 54, 1938-1944. doi: 10.1212/wnl.54.10.1938

Ye, J. C., Tak, S., Jang, K. E., Jung, J., and Jang, J. (2009). NIRS-SPM: statistical parametric mapping for near-infrared spectroscopy. Neuroimage 44, 428-447. doi: 10.1016/j.neuroimage.2008.08.036

Yu, N., Estevez, N., Hepp-Reymond, M. C., Kollias, S. S., and Riener, R. (2011). fMRI assessment of upper extremity related brain activation with an MRIcompatible manipulandum. Int. J. Comput. Assist. Radiol. Surg. 6, 447-455. doi: $10.1007 / \mathrm{s} 11548-010-0525-5$

Conflict of Interest Statement: The authors declare that the research was conducted in the absence of any commercial or financial relationships that could be construed as a potential conflict of interest.

Received: 08 October 2013; accepted: 21 January 2014; published online: 06 February 2014.

Citation: Chang P-H, Lee S-H, Gu GM, Lee S-H, Jin S-H, Yeo SS, Seo JP and Jang SH (2014) The cortical activation pattern by a rehabilitation robotic hand: a functional NIRS study. Front. Hum. Neurosci. 8:49. doi: 10.3389/fnhum.2014.00049 This article was submitted to the journal Frontiers in Human Neuroscience. Copyright (c) 2014 Chang, Lee, Gu, Lee, Jin, Yeo, Seo and Jang. This is an open-access article distributed under the terms of the Creative Commons Attribution License (CC BY). The use, distribution or reproduction in other forums is permitted, provided the original author(s) or licensor are credited and that the original publication in this journal is cited, in accordance with accepted academic practice. No use, distribution or reproduction is permitted which does not comply with these terms. 